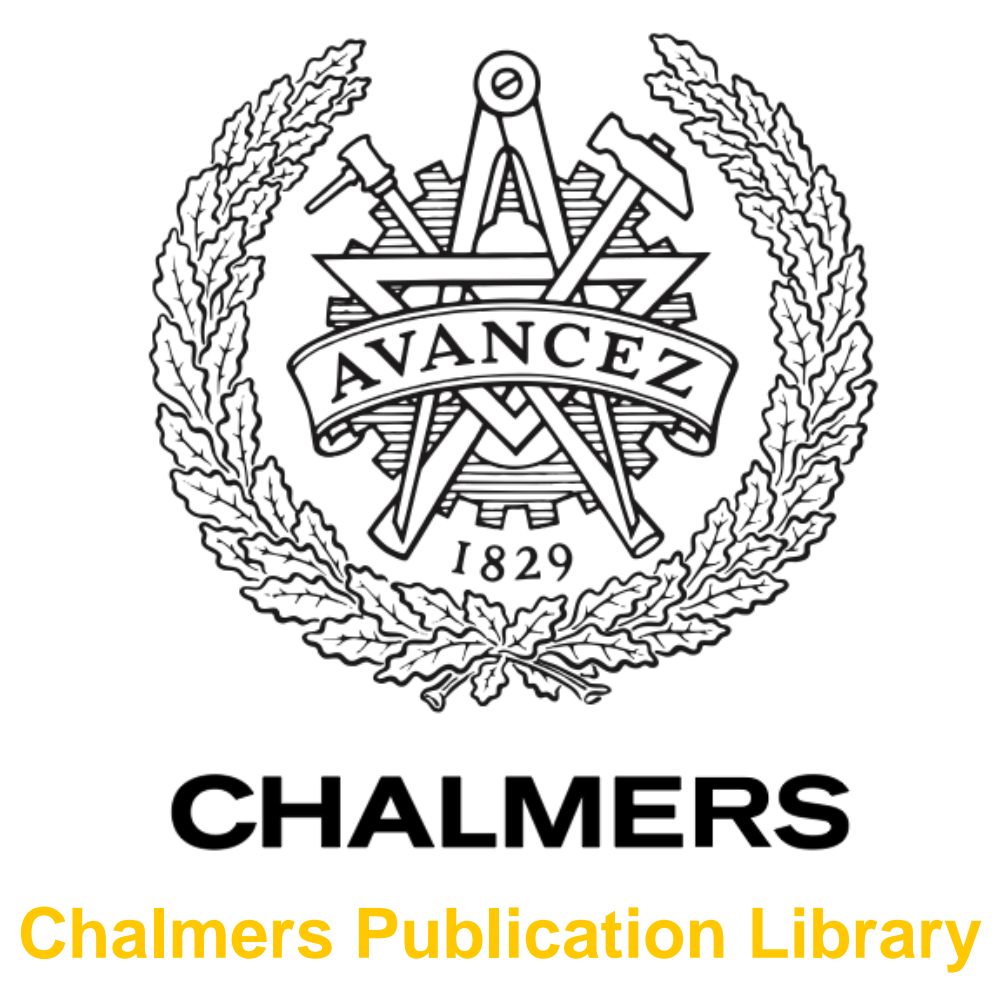

\title{
Combining scanning haptic microscopy and fibre optic Raman spectroscopy for tissue characterization
}

This document has been downloaded from Chalmers Publication Library $(\mathrm{CPL})$. It is the author's version of a work that was accepted for publication in:

Journal of Medical Engineering \& Technology (ISSN: 0309-1902)

Citation for the published paper:

Candefjord, S. ; Murayama, Y. ; Nyberg, M. (2012) "Combining scanning haptic microscopy and fibre optic Raman spectroscopy for tissue characterization". Journal of Medical

Engineering \& Technology, vol. 36(6), pp. 319-327.

http://dx.doi.org/10.3109/03091902.2012.687035

Downloaded from: http://publications.lib.chalmers.se/publication/163406

Notice: Changes introduced as a result of publishing processes such as copy-editing and formatting may not be reflected in this document. For a definitive version of this work, please refer to the published source. Please note that access to the published version might require a subscription. 


\title{
Combining scanning haptic microscopy and fibre optic Raman spectroscopy for tissue characterisation
}

\author{
Stefan Candefjord ${ }^{1-4}$, Yoshinobu Murayama ${ }^{2,5}$, Morgan \\ Nyberg $^{1,2}$, Josef Hallberg ${ }^{1,2}$, Kerstin Ramser ${ }^{1,2}$, Börje \\ Ljungberg $^{6}$, Anders Bergh ${ }^{7}$, Olof A Lindahl ${ }^{1,2,8}$ \\ ${ }^{1}$ Department of Computer Science, Electrical and Space Engineering, Luleå \\ University of Technology, 971 87, Luleå, Sweden \\ ${ }^{2}$ Centre for Biomedical Engineering and Physics, Luleå University of Technology \\ and Umeå University, Luleå and Umeå, Sweden \\ 3 Signals and Systems, Chalmers University of Technology, 412 96, Gothenburg, \\ Sweden \\ ${ }^{4}$ MedTech West, Sahlgrenska University Hospital, Blå Stråket 7, 413 45, \\ Gothenburg, Sweden \\ ${ }^{5}$ Department of Electrical and Electronics Engineering, College of Engineering, \\ Nihon University, Fukushima, Japan \\ ${ }^{6}$ Department of Surgical and Perioperative Science, Urology and Andrology, \\ Umeå University, Umeå, Sweden \\ 7 Department of Medical Biosciences, Pathology, Umeå University, Umeå, \\ Sweden \\ 8 Department of Radiation Sciences/Biomedical Engineering, Umeå university, \\ 901 87, Umeå, Sweden \\ E-mail: stefan.candefjord@chalmers.se
}




\begin{abstract}
The tactile resonance method (TRM) and Raman spectroscopy (RS) are promising for tissue characterisation in vivo. Our goal is to combine these techniques into one instrument, to use TRM for swift scanning, and RS for increasing the diagnostic power. The aim of this study was to determine the classification accuracy, using support vector machines, for measurements on porcine tissue and also produce preliminary data on human prostate tissue. This was done by developing a new experimental setup combining micro-scale TRM scanning haptic microscopy (SHM) - for assessing stiffness on a micro-scale, with fibre optic RS measurements for assessing biochemical content. We compared the accuracy for using SHM alone versus SHM combined with RS, for different degrees of tissue homogeneity. The cross-validation classification accuracy for healthy porcine tissue types using SHM alone was $65-81 \%$, and when RS was added it was increased to $81-87 \%$. The accuracy for healthy and cancerous human tissue was $67-70 \%$ when only SHM was used, and increased to $72-77 \%$ for the combined measurements. This shows that the potential for swift and accurate classification of healthy and cancerous prostate tissue is high. This is promising for developing a tool for probing the surgical margins during prostate cancer surgery.
\end{abstract}

Keywords: tactile resonance method, Raman spectroscopy, prostate cancer, support vector machines, tissue characterisation

Submitted to: Journal of Medical Engineering $\&$ Technology

$\begin{array}{ll}\text { Abbreviations } \\ \kappa & \text { curvature parameter } \\ E & \text { Young's modulus } \\ \text { MTS } & \text { micro tactile sensor } \\ \text { PZT } & \text { lead zirconate titanate } \\ \text { PBS } & \text { phosphate buffered saline } \\ \text { RBF } & \text { radial basis function } \\ \text { ROI } & \text { region of interest } \\ \text { RS } & \text { Raman spectroscopy } \\ \text { SHM } & \text { scanning haptic microscopy } \\ \text { SVM } & \text { support vector machines } \\ \text { TRM } & \text { tactile resonance method }\end{array}$

\title{
1. Introduction
}

Surgical removal of the prostate is the recommended treatment for patients suffering from high-risk localised prostate cancer [1]. To minimise the risk for cancer recurrence it is important that all cancerous tissue is removed [2]. This is a challenge for the surgeons, because minimal nerve damage is imperative for recovery of erectile function, and they have no instrument for probing the surgical margins [3]. Cancer 
can generally not be detected by the eye. Therefore, there is a great need for a tool that could detect remaining cancer [3]. The tactile resonance method (TRM) and Raman spectroscopy (RS) are two techniques that are promising for in vivo characterisation of prostate tissue $[4,5]$. Each has certain disadvantages that limit its usefulness. Our research focuses on combining these two methods to benefit from their strengths while minimising their disadvantages.

TRM mimics the perception of palpation and gives an objective measure of stiffness. It is based on the principle that the contact impedance of a vibrating rod changes when it is applied against an additional load. A change of the vibrational frequency is required to keep the phase shift constant during an application of the sensor to a sample. The frequency shift is proportional to the density and Young's modulus $(E)$ of the sample [6]. The TRM can be used with a variety of sensor sizes. For medical applications, TRM sensors have been developed with diameters ranging from 1.0 to $5.0 \mathrm{~mm}[4,6]$. A recent development has been the invention of scanning haptic microscopy (SHM), which uses a micro tactile sensor (MTS) [7]. The MTS can be made with sensor tips ranging from $0.1 \mu \mathrm{m}$ to $1.0 \mathrm{~mm}$. This has made it possible to visualise the elasticity distribution over the surface of sliced tissues of porcine heart muscle [8], porcine vascular tissues $[9,10]$ and human prostate [11], on a micro scale.

RS is a powerful technique for probing the biochemical contents of native tissue [12]. Its principle is to expose the tissue to strong monochromatic light from a laser to make molecular bonds vibrate. The Raman scattered photons obtain a wavelength shift corresponding to the difference between the initial and final vibrational energy levels. Every molecule has a unique set of vibrational frequencies, hence every tissue type gives rise to a unique Raman spectrum. Numerous in vitro studies have attained high accuracies for identifying cancerous tissue using RS [12]. However, development of clinical applications has been delayed due to a lack of adequate fibre optic probes [13]. The main disadvantages of $\mathrm{RS}$ are the shallow penetration depth of current fibre optic probes [14], that surrounding light interferes with the signal, and that strong laser illumination may harm the tissue [15].

By combining TRM and RS we can make use of the fast and gentle scanning capability of TRM to localise lesions suspected to be cancerous. These can then be probed by RS, and a diagnostic algorithm can be used to determine the pathology from the combined data.

Support vector machines (SVM) can be used for reliable tissue classification. They are collections of algorithms used for data analysis and pattern recognition [16]. The method has been used on RS data to classify different types of tissues and to detect cancer [17]. The SVM algorithm calculates the hyperplane that maximises the separation between groups. It maps the data set into a higher-dimensional space to achieve nonlinear separation, and it works well on data sets with many variables. Therefore, we here apply SVM to the multitude of variables generated from the combination of TRM and RS. 
An important step towards an in vivo application is to develop an efficient tissue classification procedure. In a previous study [4], we combined TRM and $\mathrm{RS}$ measurements using a TRM probe with $\sim 20 \mathrm{~mm}^{2}$ sensing area. Further investigations on a smaller scale were suggested. The aim of this study was to investigate the accuracy of characterisation of porcine prostate tissue, and also produce preliminary data on human prostate tissue, by developing a new experimental setup combining SHM and fibre optic RS and apply SVM on the combined data for the first time.

\section{Material and methods}

\subsection{Sample preparation}

The prostate gland was removed from eight, healthy, approx. six-month-old boars that were slaughtered at the local abattoir. A veterinarian removed the prostates with attached urethras directly after slaughter. They were enclosed in plastic bags and refrigerated until transportation. Five tissue samples were cut from each prostate. They were covered with tissue freezing medium (TFM-5 (red), Triangle Biomedical Sciences Inc., Durham, USA) and immersed in liquid nitrogen for at least $30 \mathrm{~s}$. After the samples had been frozen three holes $(\varnothing=1 \mathrm{~mm})$ were drilled as position landmarks. Each sample was cut into two $500 \mu \mathrm{m}$ thick sections using a microtome (Cryo-Star HM 560 M, Microm, Walldorf, Germany). Adjacent to both sides of each section a specimen of $5 \mu \mathrm{m}$ thickness was cut, which was stained for histologic analysis with hematoxylin and eosin according to standard procedure. The $500-\mu \mathrm{m}$ sections were stored in $-80^{\circ} \mathrm{C}<6$ months, until measurements were performed. Prior to measurements they were short-term stored in the freezer at the biomedical laboratory $\left(-20^{\circ} \mathrm{C}\right)$. All the histology specimens were scanned using a scanning microscope (ScanScope CS, 20× objective, Aperio, Vista, CA, USA).

Samples from four of the prostates were used for tests to optimise the measurement procedure. Ten arbitrary samples were taken from each of the four remaining prostates. Half were used for further optimisation tests, leaving 21 samples for the final measurements. To minimise tissue degradation the prostates were kept in sealed containers and refrigerated until snap-freezing. Furthermore, the protective fascia surrounding the prostates was removed just before the snapfreezing. All samples were snap-frozen within two days after slaughter.

A 67-year old man with prostate cancer of Gleason score seven and stage pT3 had his prostate surgically removed. One 5-mm and one 8- $\mathrm{mm}$ punch were used to take four samples from the prostate in regions suspected to contain both healthy and cancerous tissue. The samples were snap-frozen in liquid nitrogen within 30 minutes after surgery. The preparation followed the same procedure as for the porcine samples. Only three of the $500-\mu \mathrm{m}$ sections contained cancerous tissue and were included in the study.

Permission to use animal samples was obtained from the Swedish Board of 
Agriculture. The study was approved by the ethics committee of Umeå University. The participating patient gave informed written consent.

\subsection{Scanning haptic microscopy}

Topography and elasticity mapping of the 500- $\mu$ m tissue sections were measured with SHM, which uses an MTS to scan the surface and measure the elasticity distribution. The basic system configuration and its operation are well described in previous studies $[9,10]$. The system was originally developed by Murayama et al [8] and is currently available as a customisable product named SHM (P\&M Co., Ltd., Aizuwakamatsu, Fukushima, Japan).

The main part of SHM was composed of a precise XYZ stage and a Z-axis actuator, which was attached on the Z-axis stage to manipulate the MTS. Two cameras, one for monitoring the sensor tip with high magnification, and the other for observing the sample and setting the measurement region of interest (ROI) with low magnification, were mounted on the Z-axis stage. The samples were mounted on the XY-axis stage for horizontal movement. Each axis was controlled using a very fine stepping motor with a resolution of $0.01 \mu \mathrm{m}$.

The MTS is a highly sensitive resonator-based contact impedance meter capable of estimating the elastic modulus of soft tissues in micrometer scale. It consists of a cylindrical piezoelectric transducer made of lead zirconate titanate (PZT). The PZT transmits a very weak longitudinal ultrasonic wave (frequency $100-200 \mathrm{kHz}$ ) into a glass needle, which has a spherically shaped tip that makes contact with the measurement object. A phase shift circuit is used to drive the MTS to obtain high signal-to-noise ratio. Details of the composition, the electronics, and the detection principle of the MTS are published in $[7,8,18]$. The articles show that the change in the oscillation frequency $\Delta f(\mathrm{~Hz})$ for a certain indentation or $\Delta f / \delta(\mathrm{Hz} / \mu \mathrm{m})$ is highly correlated to $E$. Four different sensors were used in this study. They consisted of PZT elements that were $15 \mathrm{~mm}$ long and $2 \mathrm{~mm}$ wide, onto which a glass needle was attached $(\varnothing=1 \mathrm{~mm}$, length $21-27 \mathrm{~mm})$. The needles had tapered ends with tip diameters between 53 and $65 \mu \mathrm{m}$. The best performance was obtained for frequencies around $200 \mathrm{kHz}(188.2-200.8 \mathrm{kHz})$.

Gelatin samples were used to calibrate $\Delta f / \delta$ to $E$ at the end of each day of measurement. Six samples (G9382 Gelatin from bovine skin Type B, SigmaAldrich, St. Lois, MO, USA) with gelatin weight percentage ranging from 3 to $25 \%$ were prepared once a week. The gelatin was solidified in 53-mm Petri dishes and stored at $5{ }^{\circ} \mathrm{C}$ for at least 90 minutes before measurement. The samples were probed by SHM within three minutes in room temperature and then replaced in the refrigerator for at least 30 minutes. Next, $E$ was determined by depressing a flatended steel $\operatorname{rod}(\varnothing=2 \mathrm{~mm})$ perpendicular to the gelatin surface and recording the reactive force. A computer-controlled translation stage (NRT150P1, three NRT100, BSC103, Thorlabs, Newton, NJ, USA) controlled the motion of the rod and recorded 
the vertical impression depth $\mathrm{W}$, which was maximally $0.14 \mathrm{~mm}$, with a step size of $0.01 \mathrm{~mm}$. The reactive force was read by a $0.01 \mathrm{~g}$ resolution electronic balance (HT-600H, Elastocon, Borås, Sweden), and a stable reading was acquired after $2 \mathrm{~s}$. $E$ was calculated according to the theory by Sneddon [19] at $W=0.09 \pm 0.05 \mathrm{~mm}$ to ensure full contact. A linear model of $E$ for all samples was fitted to the mean $\Delta f / \delta(n=100)$ value using a least-squares fit, and the equation for the resulting line was used to calculate $E$ for the corresponding tissue measurements.

\subsection{Raman spectroscopy}

A fibre optic RS probe [20] (Machida Endoscope Co., Tokyo, Japan) with an outer diameter of $0.8 \mathrm{~mm}$ was connected to an RXN1 spectroscope with a continuouswave Invictus ${ }^{\mathrm{TM}}$ diode laser at $785 \mathrm{~nm}$ (both from Kaiser Optical Systems (KOSI), Ann Arbor, MI, USA). The iC Raman ${ }^{\top M}$ software (version 2.0, KOSI) was used for spectral acquisition from 100 to $3425 \mathrm{~cm}^{-1}$. The output effect to the samples was adjusted to approximately $150 \mathrm{~mW}$, corresponding to a laser input effect of $270 \mathrm{~mW}$. The integration time was set to $7 \mathrm{~s}$. The diameter of the measurement area was estimated to be $0.3 \mathrm{~mm}$ [21]. The system was calibrated for wavelength shift and energy sensitivity using neon emission lines and a NIST traceable white-light source (HoloLab Calibration Accessory, KOSI).

\subsection{Combination of $R S$ and $S H M$}

The experimental setup and principle of measurement is shown in figure 1. The RS probe was mounted on the $\mathrm{Z}$ axis of the SHM setup. An in-house developed program written in LABVIEW ${ }^{\top M}$ (version 8.6, National Instruments, Austin, TX, USA) was used to control the system.

A spatial calibration of the setup was performed using a printed grid with a line thickness of $50 \mu \mathrm{m}$. First, the axes of the camera (LifeCam Cinema ${ }^{T M}$, Microsoft, Redmond, WA, USA) for setting the ROI were carefully aligned with the axes of the stage. Second, the image distortion was established and removed by recording the image and corresponding stage coordinates of nine grid line intersections. Third, the positions of the MTS and RS probes relative to the camera were determined by using a line intersection as reference mark. The high magnification camera (DinoLite AM413TL, AnMo Electronics Corp., Hsinchu, Taiwan) of the SHM setup was used to position the probes precisely above this mark. Because the RS probe was large compared to the mark an RS mapping was conducted to improve the accuracy. A cluster analysis of the spectra clearly showed the exact location of the mark. The probe position accuracy using the camera to set the ROI was tested after calibration and was of the order of $100 \mu \mathrm{m}$. The centre-to-centre distance between the MTS and RS measurement areas was estimated to be within $20 \mu \mathrm{m}$.

After the samples were removed from the freezer they were put on top of a filter paper soaked with PBS, which in turn was mounted on a microscope slide with 


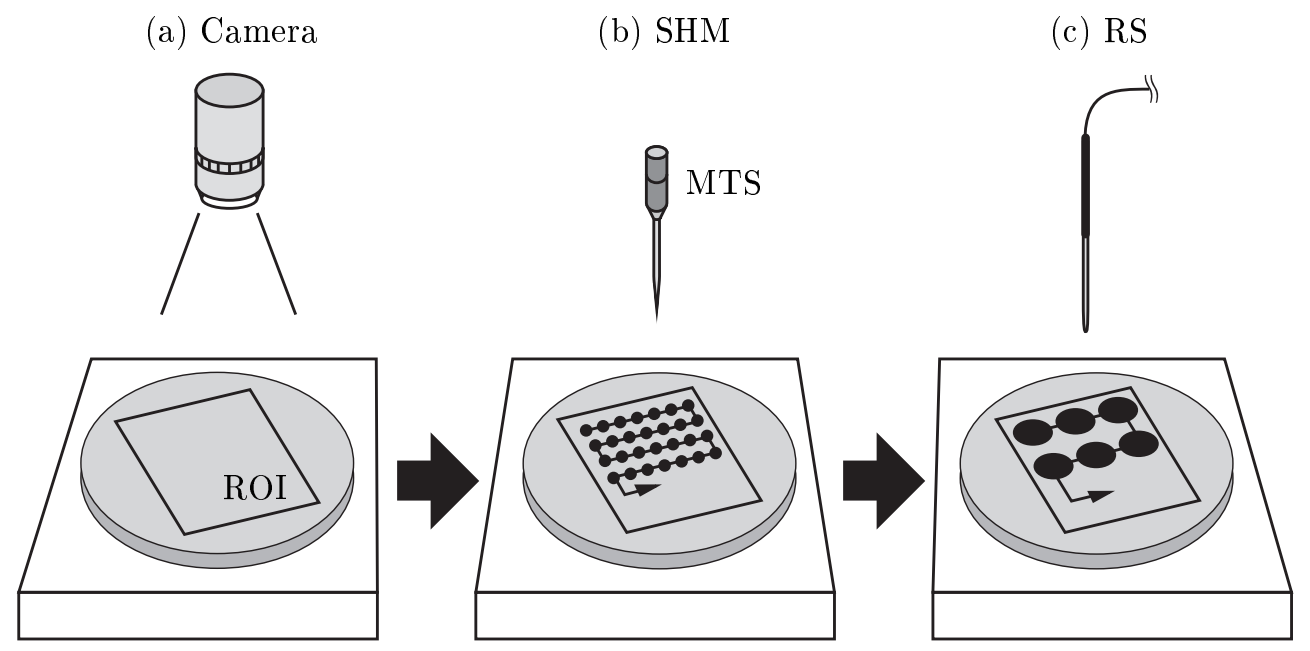

Figure 1. A flow chart illustrating the measurement scheme: (a) Setting the ROI, (b) SHM mapping of topography and elasticity, (c) RS mapping of the biochemical contents.

low Raman background (Calcium Fluoride UV grade, Crystran Ltd., Poole, UK). A simplified humid chamber was made by putting a rolled-up piece of a Kimwipe paper (Kimtech Science Kimwipes, Kimberly-Clark Inc., Roswell, GA, USA) around the sample. A pump system (Nemesys, cetoni GmbH, Korbussen, Germany) was used to supply a constant flow of PBS to the Kimwipe during the measurements. A flow of $0.23 \mu \mathrm{L} \mathrm{s}^{-1}$ was used. The distance from the RS probe to the sample was continuously adjusted to be kept constant at $400 \mu \mathrm{m}$, which provides high spectral quality [20], by using the topographic information acquired with SHM.

The ROI was chosen to be $3 \times 3 \mathrm{~mm}$ for all measurements on porcine prostate. $60 \times 60=3600$ measurement points were acquired with SHM using a $50-\mu \mathrm{m}$ step size, and subsequently $10 \times 10=100$ measurement points were acquired with RS using a $300-\mu \mathrm{m}$ step size. The step sizes were chosen to match the diameters of the measurement areas. Measurements were started 15 minutes after the samples were removed from the freezer. The measurement time was less than 45 minutes, of which the SHM took approx. 30 minutes. The same measurement procedure was used for the human samples. However, the ROI was adjusted to the sample size and positioned to cover the regions with cancerous tissue. A total of 275 spectra and 9900 SHM measurement points were acquired from the human samples.

\subsection{Data preprocessing and analysis}

MATLAB $^{\circledR}$ (version R2011b including Statistics Toolbox version 7.6, MathWorks Inc., Natick, MA, USA) was used for all preprocessing and analysis. The LIBSVM [22] library interface to MATLAB ${ }^{\circledR}$ (version 3.11) was used for the SVM analysis. Algorithms not available in MATLAB ${ }^{\circledR}$ were written in-house. Eilers' algorithm [23] was available for MATLAB ${ }^{\circledR}$ via the Internet. Two separate analyses 
were conducted, one for the porcine samples and one for the human samples.

2.5.1. Histologic analysis The scans of the tissue specimens were randomly assigned and histologically analysed by two operators (authors S.C. and M.N.). The area of measurement was localised by comparing the histology image to the picture of the measured sample and to the stiffness map acquired by the SHM. Using Photoshop ${ }^{\circledR}$ (version CS5, Adobe ${ }^{\circledR}$, San Jose, CA, USA) the histology image was rotated, scaled and sometimes skewed so the drill holes and the morphological features overlapped. A grid with $50 \mu \mathrm{m}$ spacing that defined the SHM measurement points was then superimposed over the histology image. The tissue type of each square of the grid was manually determined. For porcine prostate there were three main tissue types: epithelium, lumen and stroma. For human prostate there were six main tissue types: normal epithelium, cancerous epithelium, lumen, prostate stones, normal stroma and cancerous stroma. Points that could not be determined were excluded from the analysis. The quality of the histologic analysis was ascertained by a board certified pathologist (author A.B.), with over 25 years of experience of prostate histopathology. There was a relatively large uncertainty in the localisation of the measurement area. Therefore, we performed the analysis of SHM data on more homogeneous tissue regions by extracting the subset of measurement points whose neighbours were of the same tissue type.

2.5.2. $S H M$ The stiffness indicator $\Delta f / \delta$, and an additional curvature parameter $\kappa\left(\mathrm{Hz} \mu \mathrm{m}^{-2}\right)$, was calculated from the frequency shift data. The threshold for surface detection was set to $10 \mathrm{~Hz}$, and only a shift in the direction expected for the current sensor triggered detection. A linear least-squares fit was performed to the five consecutive data points starting from the point of surface detection. A second-degree polynomial was fitted to the same data points. The coefficient for the quadratic term, $\kappa$, was a measure of the curvature of the frequency-indentation curve, and was related to nonlinear effects. Measurements for which $<3$ data points were available, or for which the coefficient of determination $R^{2}<0.9$ for the linear fit, were discarded.

2.5.3. $R S$ For the porcine samples the raw Raman spectra were mainly composed of signals from the tissue, the optic fibres in the RS probe [20], and the filter paper on which the sample lay. To extract the useful Raman signal a number of consecutive preprocessing steps were applied to the raw spectra:

(i) The Fingerprint spectral interval $600-1800 \mathrm{~cm}^{-1}$ was selected [12].

(ii) The background generated by the RS probe itself was estimated by measuring on a microscope slide with low inherent background. It was then removed from the raw spectra by applying the variance minimisation method [24].

(iii) Light smoothing was employed using Eilers' algorithm with $d=2$ and $\lambda=$ $10[23]$. 
(iv) The filter paper generated a number of sharp Raman peaks, which were subtracted using the second-derivative variance minimisation method [24].

(v) The fluorescence background was subtracted by fitting a piecewise polynomial to each spectrum [25].

(vi) The spectra were vector normalised so that their integrated intensities were equalised.

Spectral peaks were tentatively assigned to specific molecular vibrations by using the database [26].

For the human samples the Fingerprint interval could not be used due to strong fluorescence caused by the ink applied to the surgical resection margin. Therefore, the interval 2400-3425 $\mathrm{cm}^{-1}$ was selected, which has been shown to be rich in diagnostic information [27]. Spectra saturated within this interval were removed $(n=30)$ from the analysis. The raw spectra were smoothed $(d=2$ and $\lambda=100)$, background-reduced, and normalized using the algorithms mentioned above.

2.5.4. Classification We determined the tissue type of each RS measurement area, which was $0.3 \mathrm{~mm}$ in diameter, by assigning it to the type most abundant within the area. The tissue types for the human samples were grouped into either healthy or cancerous tissue. Areas that did not contain more than a threshold percentage of the same tissue type were excluded. Analyses were performed for three thresholds: $50 \%, 67 \%$ and $83 \%$. The preprocessed RS data, the mean stiffness of each area, and the mean value of $\kappa$ for each area, were input to the SVM. Each input variable was scaled to the interval [ $\left[\begin{array}{ll}0 & 1\end{array}\right]$ to give all attributes equal importance regardless of numeric range. To manage the unbalanced data sets, weights were assigned to each tissue type in proportion to their total abundance.

The approach for SVM classification suggested by Chen et al [28] with the radial basis function $(\mathrm{RBF})$ kernel was adopted. This approach suggests that the kernel parameter $\gamma$ and an additional penalty parameter $C$, which controls the tradeoff between complexity of the decision rule and frequency of error [16], can be decided through a grid search in which the highest cross-validation accuracy is found. Crossvalidation is used to prevent overfitting the classifier to the training data. The data set is randomly divided into a number of subsets, five is recommended by Chen et al [28] and was used in this study. Each subset in turn is used as validation data and the other subsets combined form the training data. The process is repeated until each subset has been used as validation data, and the overall accuracy can then be determined. Intervals from $2^{-15}$ to $2^{3}$ and from $2^{-5}$ to $2^{15}$ were searched to determine the RBF width $\gamma$ and the penalty factor $C$, respectively. A too high value of $C$ would overfit the support vectors, and a too low value would underfit them. 
2.5.5. Statistics The Lillie test was used to evaluate data normality. The KruskalWallis nonparametric test followed by the Tukey-Kramer multiple comparison test were used to assess significant differences in stiffness and $\kappa$ values between the tissue types. A $p$-value less than 0.05 was considered statistically significant.

\section{Results}

The data analysis was conducted on all three human samples and 14 of the 21 porcine samples. Seven had to be excluded for the following reasons: to avoid damage to the MTS, due to tissue adsorption on the MTS tip, or due to an inappropriate MTS driving frequency. For three samples the measurement area could not be identified on the histologic image.

\section{1. $S H M$}

The calibration measurements on gelatin showed that the stiffness indicator was linear to $E\left(R^{2} \geq 0.99\right)$. Figure 2 shows a typical example of an elasticity mapping. Tissue structures were clearly observed as differences in their elasticity (figure 2(c)), where stroma corresponded to high elasticity regions and lumen mostly to low elasticity regions.

For the porcine samples the median values of $E$ and $\kappa$ for epithelium, lumen and stroma were significantly different $(p<0.05)$. There were relatively large overlaps of the ranges of $E$ and $\kappa$ for the tissue types (figures 3 and 4 ). The stiffness data was not normally distributed $(p<0.05)$, a positive skewness was observed. $\kappa$ did not follow a normal distribution.

For the human samples there were significant differences between the median $E$ and $\kappa$ values $(p<0.05)$. Lumen was significantly softer than all other groups, except for healthy epithelium $(p<0.05)$. Prostate stones were significantly stiffer than cancerous epithelium, healthy stroma, and lumen $(p<0.05)$. The median $\kappa$ value for cancerous stroma was significantly higher than all other groups, except for prostate stones. Furthermore, $\kappa$ was higher for cancerous epithelium than lumen and healthy epithelium $(p<0.05)$. The median values of $E$ and $\kappa$ are shown in figure 5 . There were large overlaps between the groups. $E$ and $\kappa$ were generally not normally distributed within the groups.

\section{2. $R S$}

The mean spectra of the porcine tissue types showed the following differences (figure 6):

- Changes in the collagen content seen in peaks at $1654 \mathrm{~cm}^{-1}$ (Amide I), $1448 \mathrm{~cm}^{-1}$ (Amide III), $935 \mathrm{~cm}^{-1}$ and $813 \mathrm{~cm}^{-1}$. Stroma showed the highest collagen content and lumen the lowest. 
(a) Histology image

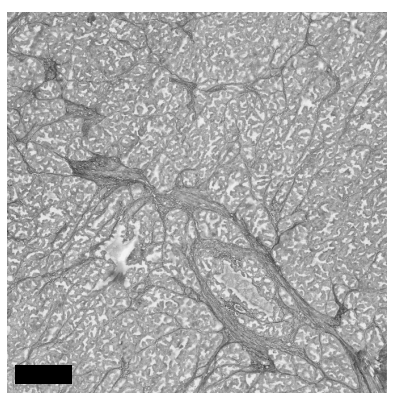

(b) Histologic analysis

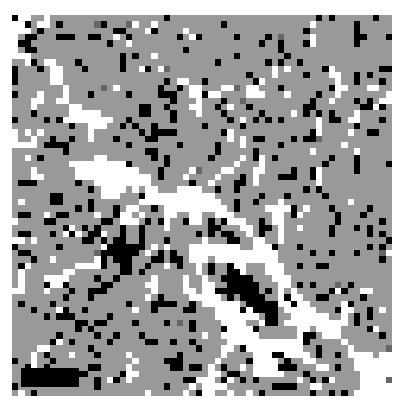

(c) Stiffness

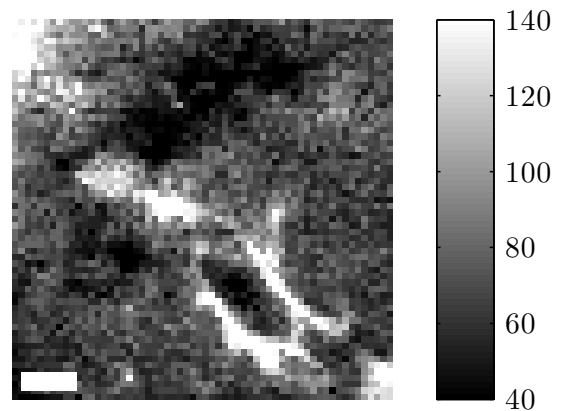

Figure 2. In the histologic analysis lumen is black, epithelium grey and stroma white. The scale bars show $500 \mu \mathrm{m}$.

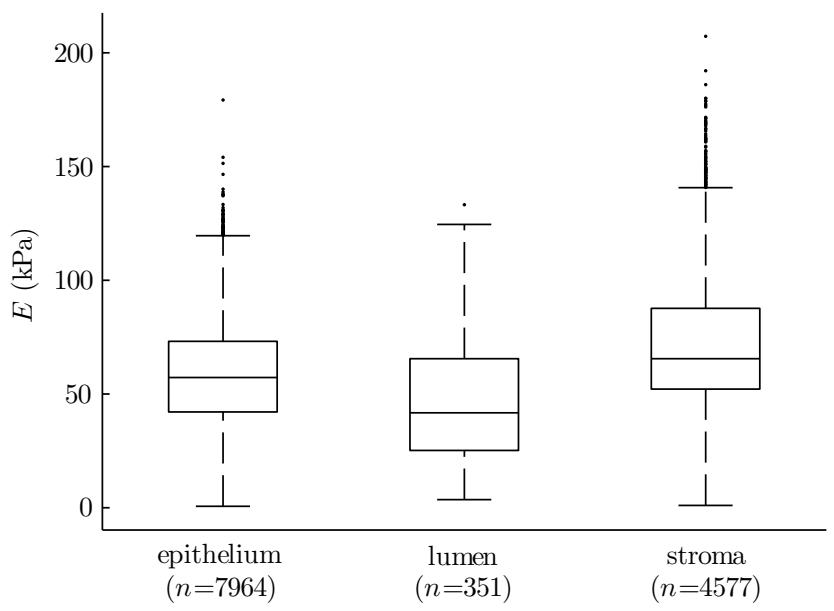

Figure 3. A box plot of the $E$ values for the different porcine tissue types. The line in the middle of the box shows the median, and the bottom and the top of the box show the 25 th and 75 th percentile, respectively. The whiskers extend to 1.5 times the interquartile range away from the top or bottom of the box, or to the furthest observations from the box. Data points outside the whiskers are plotted individually.

- Changes in the DNA content seen in the region $640-850 \mathrm{~cm}^{-1}$. Stroma showed the highest DNA content and lumen the lowest.

- Changes in the lipid and fat content seen in the peaks between 1720 to $1790 \mathrm{~cm}^{-1}$. Lumen showed the highest content.

For the human samples it was found that the blue ink applied to the surgical resection margin, which is standard clinical procedure, caused a very strong fluorescent signal that often saturated the CCD detector and swamped the signal from the tissue in the Fingerprint interval (figure 7). However, the fluorescence decreased towards the high wavenumber region. 


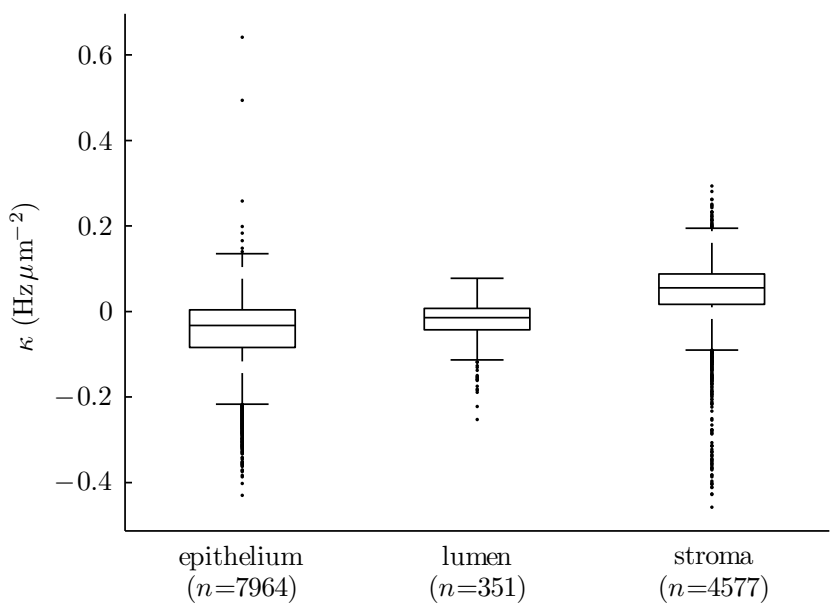

Figure 4. A box plot of the $\kappa$ values for the different porcine tissue types. The line in the middle of the box shows the median, and the bottom and the top of the box show the 25 th and 75 th percentile, respectively. The whiskers extend to 1.5 times the interquartile range away from the top or bottom of the box, or to the furthest observations from the box. Data points outside the whiskers are plotted individually.

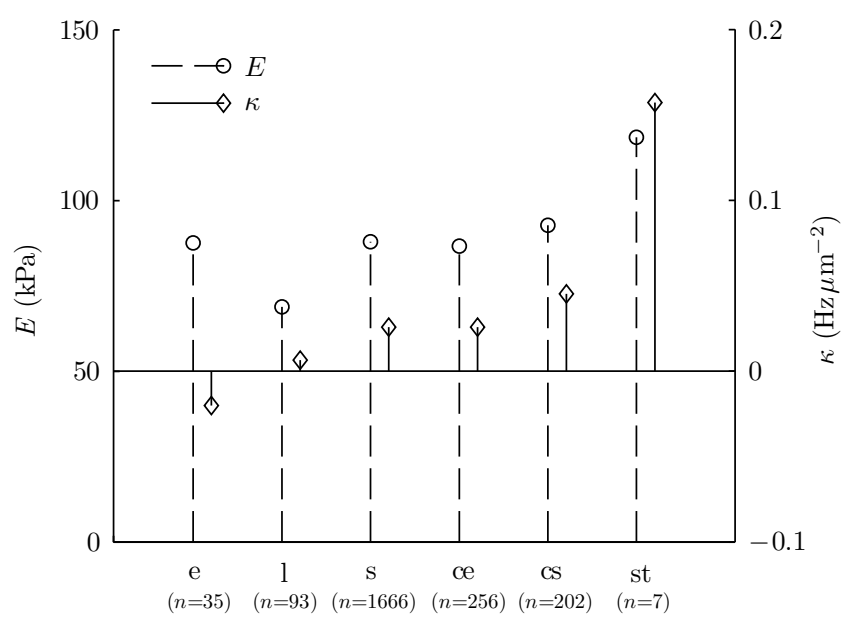

Figure 5. The medians of the $E$ and $\kappa$ values for the human tissue types. $\mathrm{e}=$ epithelium, $\mathrm{l}=$ lumen, $\mathrm{s}=$ stroma, $\mathrm{ce}=$ cancerous epithelium, $\mathrm{cs}=$ cancerous stroma, and $\mathrm{st}=$ prostate stones.

\subsection{Classification}

Table 1 shows the classification results for porcine tissue, and table 2 shows the results for human tissue. The cross-validation accuracy was generally increased for the more homogeneous tissue regions. Furthermore, by adding RS to SHM the accuracy was substantially increased. The values of $C$ and $\gamma$ ranged from 1.3 to 33000 and 0.015 to 7.0 , respectively, for porcine samples. For human tissue $C$ was between 140 and 33000, and $\gamma$ was between 0.0017 and 7.7. 


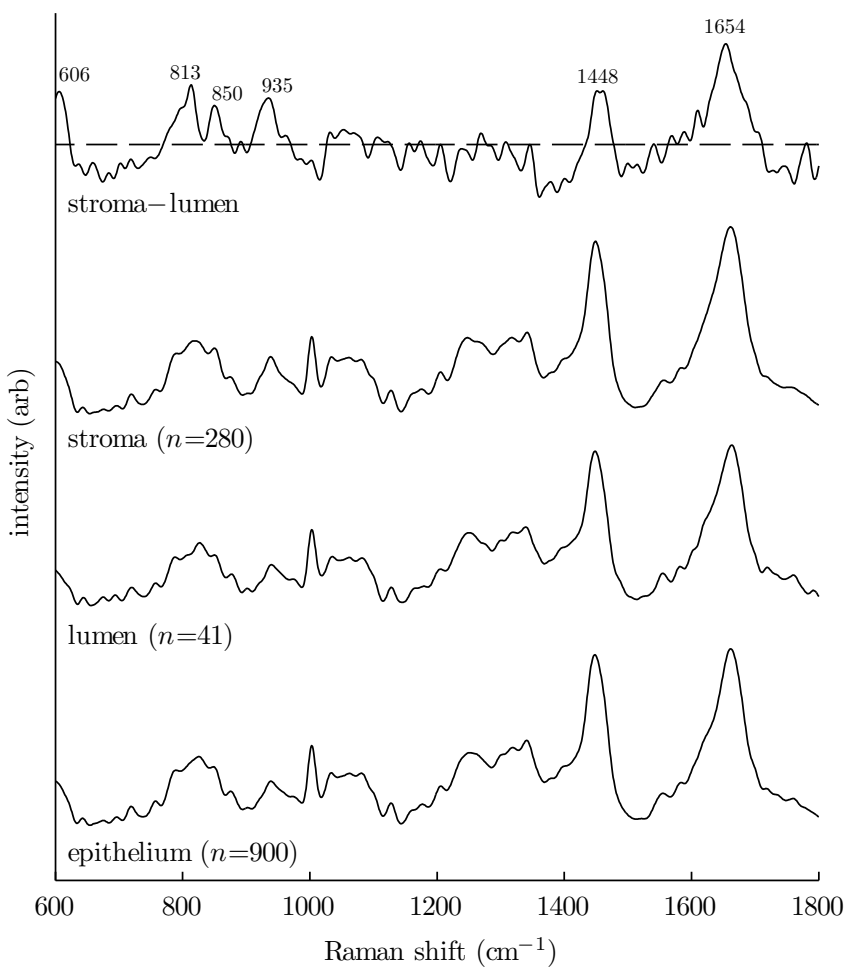

Figure 6. The mean Raman spectra of the porcine tissue types. A difference spectrum between stroma and lumen, which differed the most, is shown at the top. Its intensity has been multiplied by four to enhance the differences. arb $=$ arbitrary units.

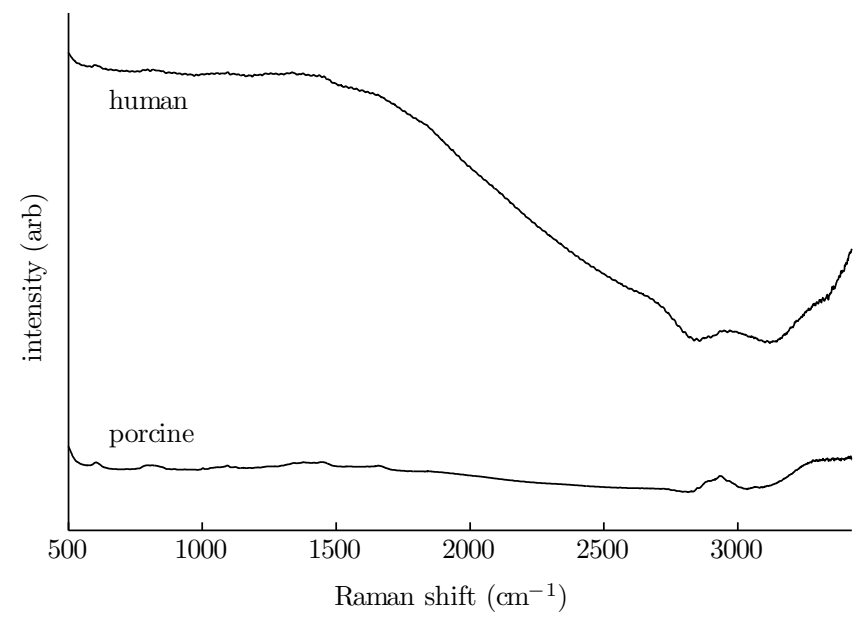

Figure 7. The mean untreated spectra of all human and porcine measurements, showing the magnitude of the ink-induced fluorescence in the human samples. arb = arbitrary units. 
Table 1. SVM classification accuracies for determining porcine tissue types from SHM and RS data. CVA = cross-validation accuracy, $\mathrm{PA}=$ prediction accuracy.

\begin{tabular}{|c|c|c|c|c|c|c|}
\hline \multirow[t]{2}{*}{ Homogeneity threshold } & \multicolumn{2}{|c|}{$>50 \%(n=1221)$} & \multicolumn{2}{|c|}{$>67 \%(n=926)$} & \multicolumn{2}{|c|}{$>83 \%(n=519)$} \\
\hline & CVA & $\mathrm{PA}$ & CVA & $\mathrm{PA}$ & CVA & $\mathrm{PA}$ \\
\hline SHM & 64.9 & 66.4 & 69.4 & 70.4 & 80.7 & 84.6 \\
\hline $\mathrm{RS}$ & 80.2 & 100 & 82.9 & 100 & 86.9 & 96.3 \\
\hline $\mathrm{SHM}+\mathrm{RS}$ & 80.8 & 99.7 & 83.2 & 99.7 & 87.3 & 96.1 \\
\hline
\end{tabular}

Table 2. SVM classification accuracies for distinguishing healthy and cancerous human prostate tissue. $\mathrm{CVA}=$ cross-validation accuracy, $\mathrm{PA}=$ prediction accuracy.

\begin{tabular}{|c|c|c|c|c|c|c|}
\hline \multirow[t]{2}{*}{ Homogeneity threshold } & \multicolumn{2}{|c|}{$>50 \%(n=204)$} & \multicolumn{2}{|c|}{$>67 \%(n=190)$} & \multicolumn{2}{|c|}{$>83 \%(n=178)$} \\
\hline & CVA & $\mathrm{PA}$ & CVA & $\mathrm{PA}$ & CVA & $\mathrm{PA}$ \\
\hline $\mathrm{SHM}$ & 69.6 & 73.5 & 67.4 & 71.6 & 66.9 & 72.5 \\
\hline $\mathrm{RS}$ & 71.6 & 100 & 74.7 & 100 & 77.0 & 100 \\
\hline $\mathrm{SHM}+\mathrm{RS}$ & 71.6 & 100 & 76.8 & 97.4 & 77.0 & 100 \\
\hline
\end{tabular}

\section{Discussion}

This study presents a new measurement setup capable of obtaining spatially resolved $\mathrm{SMH}$ and RS data from the same locations, for investigations of relationships between biochemical content and stiffness, which may be of interest in a broad range of biomedical applications. By using the topographic information from SHM the system enables RS scanning of samples with uneven surfaces. In this study we measured porcine and human prostate tissue, and applied SVM to classify healthy and cancerous tissue from the combined SHM and RS data. The combined classification accuracy was $81-87 \%$ for healthy porcine tissue, and $72-77 \%$ for healthy and cancerous human tissue, depending on the homogeneity of the tissue. We have shown that the classification accuracy for using SHM alone is good, and by adding RS to SHM the classification accuracy is substantially increased.

This study confirms the results of Jalkanen et al [29,30] and Eklund et al [31]. They used TRM sensors with diameters of 5 and $2 \mathrm{~mm}$, respectively, whereas this study mapped the elasticity on a micrometer scale. They concluded that healthy stroma was stiffer than glandular tissue, i.e. lumen and epithelium, and that prostate stones increased tissue stiffness. Furthermore, Jalkanen et al [30] showed that cancerous prostate tissue was significantly stiffer than glandular tissue $(p<0.05)$, whereas stroma and prostate stones could not be distinguished from cancer. This agrees well with our findings, which in addition indicate that cancerous 
epithelium and healthy stroma are the most difficult tissue types to distinguish, whereas cancerous stroma shows increased stiffness (figure 5). Tuxhorn et al [32] observed an elevated amount of collagen and loss of smooth muscle in cancerous stroma, which is a possible explanation for the increased stiffness observed in this study. Using SHM, Murayama et al [11] mapped the elasticity of human prostate and observed that the cancerous node (about $100 \mu \mathrm{m}$ in diameter) was stiffer than surrounding normal tissues. However, it was technically difficult to compare the elasticity map to the histology image. Thus, the elasticity distribution could not be further analysed [11].

In this study, the localisation of the measurement area on the histology images was done manually. The histology specimens were often deformed, which made it more difficult to overlap the morphological features of the histologic images and the stiffness maps. Since the step size of the SHM mappings was merely $50 \mu \mathrm{m}$, a slight localisation error would have caused many measurement points to be misclassified. To minimise the impact of this, we conducted the analysis of SHM data on homogeneous tissue regions. We observed that the separation of $E$ for the tissue types then increased, and the deviations from normality decreased. This confirmed that many points had been misclassified. For the SVM analysis the measurement areas were $300 \mu \mathrm{m}$ in diameter, so the localisation errors did not affect the outcome as much. Still, higher classification accuracy may have been attained with smaller localisation errors. In future studies an objective and more accurate localisation may be provided by developing an algorithm that can assist in identifying the measurement area.

Histology specimens from both above and below the measurement sample were acquired to give a rough estimate of the tissue distribution across the sample. The specimens that were cut below the measurement samples were of low quality. We were only able to conclude that the cancerous tissue to some extent continued across the human 500- $\mu \mathrm{m}$ sections. Thus, the histologic analysis conveyed information only about the tissue type at the topmost layer of the tissue. The probes picked up signal from deep-laying layers of tissue. The Raman spectra even contained peaks from the filter paper under the tissue. Jalkanen et al [30] showed that the TRM was sensitive to layers of tissue lying deeper than the impression depth in a weighted tissue proportion model. In this study, it can therefore be suspected that since the MTS was impressed about $40 \mu \mathrm{m}$, it also sensed even deeper layers.

Maintaining tissue moistness is fundamental for SHM measurements [10, 33]. In this study we used a simple but effective method in which the sample was placed on a filter paper and surrounded by a shelter to which a constant flow of PBS was supplied. Extensive tests were carried out to find an optimal amount of flow to prevent tissue dehydration during the total SHM measurement time of approx. 30 minutes. Six repeated SHM mappings were acquired on the same area of $10 \times 10$ points on porcine prostate tissue during 30 minutes. The optimal flow was determined by minimising the change in mean stiffness while maximising the 
correlation coefficients between the maps. In the final SHM mappings no general trend of sample dehydration was observed in plots of $E$ versus acquisition time. However, dehydration/flooding may have contributed to the large spread of $E$. In future studies the stability of the mappings and the quality of the data may be enhanced by using a moisture chamber, or immersing the sample fully in PBS [10].

Gelatin was chosen as material for stiffness calibration because it is a biological material easily prepared in a stiffness range comparable to that of prostate tissue. A potential disadvantage of gelatin is that its stiffness is temperature dependent. This effect was suppressed by storing the samples in a refrigerator and minimising the time in room temperature to less than three minutes. No temperature effect was observed in the SHM data. For two of the porcine prostate samples the gelatin calibration from the previous day was used to calculate $E$ because the MTS tip was broken after the tissue measurement due to operator mistake.

The choice of using SVM for classification was made based on the amount of variables and data, as well as the likelihood of non-separable classifications. SVM is well suited to handle this type of data by using soft margins to deal with nonseparable classifications [16]. Furthermore, by using kernel functions the SVM is able to handle a large amount of variables and large quantities of data. The classification accuracy of SVM depends on the kernel function $k\left(x_{i}, x_{j}\right)$. Several of the non-linear kernels were tested, including the inhomogeneous polynomial kernel of the form $k\left(x_{i}, x_{j}\right)=\left(x_{i} \cdot x_{j}\right)^{d}$, the RBF of the form $k\left(x_{i}, x_{j}\right)=e^{-\gamma\left\|\left(x_{i}-x_{j}\right)\right\|^{2}}, \gamma>0$, and the sigmoid kernel of the form $k\left(x_{i}, x_{j}\right)=\tanh \left(\gamma x_{i} \cdot x_{j}+r\right), \gamma>0, r<0$ where $x_{i}, x_{j}$ are data vectors and $\gamma, d$ and $r$ are kernel parameters. Tests showed that the best results were given by the RBF kernel. This agrees with Sattlecker et al [17], who compared different SVM kernels for classifying RS measurements on lymph node samples from breast cancer patients.

From tables 1 and 2 we see that by using SHM alone the cross-validation classification accuracy was $65-81 \%$. Thus, stiffness measurements are valuable for tissue characterisation and for localising stiff nodules. The nonlinear parameter $\kappa$, which was a measure of the curvature of the measurement curve, had a large positive impact on the accuracy achieved by SHM. There was a relatively large separation of the $\kappa$ values for the human tissue types, and we see from figure 5 that $\kappa$ seems too add complementary information to $E$, especially for differentiating healthy epithelium. This suggests that $\kappa$ has a diagnostic value. Figure 5 also indicates that the similarities of the $E$ and $\kappa$ values for healthy stroma and cancerous epithelium may have been the main factor limiting the accuracy for distinguishing healthy and cancerous tissue. The patient in this study suffered from prostatitis, i.e. inflammation of the prostate gland, which is a common cause of false positives in elastographic examinations of the prostate [5, 34]. The inflammation may have increased the stiffness of the healthy tissue in this patient.

The classification accuracy increased substantially when RS was added to SHM (tables 1 and 2). For the porcine samples it was over $80 \%$, even when the most 
heterogeneous tissue regions were included. For the human samples the accuracy was lower. The main reason was most likely the low quality of the Raman signal, which was quenched by the fluorescence from the ink applied to the surgical margin. Even though the fluorescence was decreased in the high wavenumber region (figure 7), the spectral quality was lower and considerably more noisy than the spectra of porcine tissue. Application of ink to the surgical margin is standard clinical procedure, and used for investigating if there is any remaining cancerous tissue in the patient after surgery [3]. Our findings show that the ink contaminates even tissue regions that are not immediately adjacent to the margin and shows no visual signs of ink.

The classification accuracy using RS alone was similar to the accuracy using SHM plus RS (tables 1 and 2). Although this indicates that for a hand-held probe incorporating both techniques TRM would not add much value to the diagnostic accuracy, the idea is to quickly scan the tissue with TRM and only use RS at suspected lesions. In addition, the TRM sensor could give tactile feedback, indicating when the surgeon touches the tissue. Whether the future diagnostic algorithm should rely on the combined information or just the RS data will be evaluated in further studies.

The high classification accuracies for combined SHM and RS measurements on heterogeneous tissue regions indicate that the proposed combined instrument will be strong for investigating tissue boundaries. Furthermore, the results indicate that the rate of misclassification for homogeneous regions will be low. This is very promising for an application where surgical margins are probed for the presence of cancer.

\section{Conclusion}

This study presents a new experimental setup for acquiring combined SHM and RS data. We show that TRM and RS can successfully be used to identify different prostate tissue types by using SVM. The results indicate accurate detection of cancerous prostate tissue. This is promising for the development of an instrument that combines these two methods for tissue characterisation during prostate cancer surgery.

\section{Acknowledgments}

This work was supported by grants from the Objective 2 Norra Norrland-EU Structural Fund and the Kempe Foundation. We wish to thank Mats Gustavsson for helping us obtaining porcine prostate samples, Kerstin Löfquist and Kerstin Stenberg at the dept. of Pathology and Cytology at Sunderby Hospital for preparing porcine histology specimens, and Kerstin Almroth, Pernilla Andersson and Birgitta Ekblom at the urology and pathology units at Norrland's University Hospital for preparing human histology specimens. 


\section{Declaration of interest}

The authors report no conflicts of interest.

\section{References}

[1] Basir Tareen, Joseph Kimmel, and William C Huang. Contemporary treatment of high-risk localized prostate cancer. Expert Rev Anticancer Ther, 10:1069-1076, 2010.

[2] Theodorus H van der Kwast, Michel Bolla, Hein Van Poppel, Paul Van Cangh, Kris Vekemans, Luigi Da Pozzo, Jean-Francois Bosset, Karl H Kurth, Fritz H Schr^der, and Laurence Collette. Identification of patients with prostate cancer who benefit from immediate postoperative radiotherapy: EORTC 22911. J Clin Oncol, 25:4178-4186, 2007.

[3] Ofer Yossepowitch, Anders Bjartell, James A Eastham, Markus Graefen, Bertrand D Guillonneau, Pierre I Karakiewicz, Rodolfo Montironi, and Franceso Montorsi. Positive surgical margins in radical prostatectomy: outlining the problem and its long-term consequences. Eur Urol, 55:87-99, 2009.

[4] Stefan Candefjord, Morgan Nyberg, Ville Jalkanen, Kerstin Ramser, and Olof A. Lindahl. Combining fibre optic Raman spectroscopy and tactile resonance measurement for tissue characterization. Meas Sci Technol, 21(125801), 2010.

[5] S. Candefjord, K. Ramser, and O. A. Lindahl. Technologies for localization and diagnosis of prostate cancer. J Med Eng Technol, 33:585-603, 2009.

[6] O. A. Lindahl, C. E. Constantinou, A. Eklund, Y. Murayama, P. Hallberg, and S. Omata. Tactile resonance sensors in medicine. J Med Eng Technol, 33:263-273, 2009.

[7] Y Murayama and S Omata. Fabrication of micro tactile sensor for the measurement of microscale local elasticity. Sensor Actuat A, 109:202-207, 2004.

[8] Y Murayama, CE Constantinou, and S Omata. Development of tactile mapping system for the stiffness characterization of tissue slice using novel tactile sensing technology. Sensor Actuat A, 120:543-549, 2005.

[9] T Oie, H Suzuki, T Fukuda, Y Murayama, S Omata, K Kanda, and Y Nakayama. Tactile mapping system: a novel imaging technology for surface topography and elasticity of tissues or organs. Innovations, 4:345-350, 2009.

[10] Tomonori Oie, Hisato Suzuki, Yoshinobu Murayama, Toru Fukuda, Sadao Omata, Keiichi Kanda, Keiichi Takamizawa, and Yasuhide Nakayama. Surface elasticity imaging of vascular tissues in a liquid environment by a scanning haptic microscope. J Artif Organs, 13:121-125, 2010.

[11] Yoshinobu Murayama, Sadao Omata, Toshikuni Yajima, Qiyu Peng, Keiichi Shishido, Donna M. Peehl, and Christos E. Constantinou. High resolution regional elasticity mapping of the human prostate. In Proceedings of the 29th Annual International Conference of the IEEE EMBS. Cit'̇ Internationale, Lyon, France. August 23-26, 200\%, pages 5802-5805, 2007.

[12] A. Mahadevan-Jansen and R. R. Richards-Kortum. Raman spectroscopy for the detection of cancers and precancers. J Biomed Opt, 1:31-70, 1996.

[13] Annieke Nijssen, Senada Koljenović, Tom C Bakker Schut, Peter J Caspers, and Gerwin J Puppels. Towards oncological application of Raman spectroscopy. J Biophotonics, 2:29-36, 2009.

[14] Pavel Matousek. Deep non-invasive Raman spectroscopy of living tissue and powders. Chem Soc Rev, 36:1292-1304, 2007.

[15] Stefan Candefjord, Kerstin Ramser, and Olof A Lindahl. Effects of snap-freezing and nearinfrared laser illumination on porcine prostate tissue as measured by Raman spectroscopy. Analyst, 134:1815-1821, 2009. 
[16] Corinna Cortes and Vladimir Vapnik. Support-Vector Networks. Machine Learning, 20:273$297,1995$.

[17] Martina Sattlecker, Conrad Bessant, Jennifer Smith, and Nick Stone. Investigation of support vector machines and Raman spectroscopy for lymph node diagnostics. Analyst, 135:895$901,2010$.

[18] Y Murayama, CE Constantinou, and S Omata. Micro-mechanical sensing platform for the characterization of the elastic properties of the ovum via uniaxial measurement. J Biomech, 37:67-72, 2004.

[19] Ian N. Sneddon. The relation between load and penetration in the axisymmetric boussinesq problem for a punch of arbitrary profile. Int J Eng Sci, 3:47-57, 1965.

[20] Yusuke Hattori, Yuichi Komachi, Toru Asakura, Toru Shimosegawa, Gen-Ichi Kanai, Hideo Tashiro, and Hidetoshi Sato. In vivo Raman study of the living rat esophagus and stomach using a micro-Raman probe under an endoscope. Appl Spectrosc, 61:579-584, 2007.

[21] Yuichi Komachi, Takashi Katagiri, Hidetoshi Sato, and Hideo Tashiro. Improvement and analysis of a micro Raman probe. Appl Opt, 48:1683-1696, 2009.

[22] Chih-Chung Chang and Chih-Jen Lin. LIBSVM: a library for support vector machines, 2001. Software available at http://www.csie.ntu.edu.tw/ cjlin/libsvm.

[23] P H Eilers. A perfect smoother. Anal Chem, 75:3631-3636, 2003.

[24] Yvette L Loethen, Dongmao Zhang, Ryan N Favors, Sara B G Basiaga, and Dor Ben-Amotz. Second-derivative variance minimization method for automated spectral subtraction. Appl Spectrosc, 58:272-278, 2004.

[25] A. Cao, A. K. Pandya, G. K. Serhatkulu, R. E. Weber, H. Dai, J. S. Thakur, V. M. Naik, R. Naik, G. W. Auner, R. Rabah, and D. C. Freeman. A robust method for automated background subtraction of tissue fluorescence. J Raman Spectrosc, 38:1199-1205, 2007.

[26] Z Movasaghi, S Rehman, and IU Rehman. Raman spectroscopy of biological tissues. Appl Spectrosc Rev, 42:493-541, 2007.

[27] S. Koljenović, T. C. Bakker Schut, R. Wolthuis, B. de Jong, L. Santos, P. J. Caspers, J. M. Kros, and G. J. Puppels. Tissue characterization using high wave number Raman spectroscopy. J Biomed Opt, 10(031116), 2005.

[28] Yi-Wei Chen and Chih-Jen Lin. Combining SVMs with various feature selection strategies. In Isabelle Guyon, Masoud Nikravesh, Steve Gunn, and Lotfi Zadeh, editors, Feature Extraction, volume 207 of Studies in Fuzziness and Soft Computing, pages 315-324. Springer Berlin / Heidelberg, 2006.

[29] Ville Jalkanen, Britt M Andersson, Anders Bergh, Börje Ljungberg, and Olof A Lindahl. Prostate tissue stiffness as measured with a resonance sensor system: a study on silicone and human prostate tissue in vitro. Med Biol Eng Comput, 44:593-603, 2006.

[30] Ville Jalkanen, Britt M Andersson, Anders Bergh, Börje Ljungberg, and Olof A Lindahl. Resonance sensor measurements of stiffness variations in prostate tissue in vitro - a weighted tissue proportion model. Physiol Meas, 27:1373-1386, 2006.

[31] A. Eklund, A. Bergh, and O. A. Lindahl. A catheter tactile sensor for measuring hardness of soft tissue: measurement in a silicone model and in an in vitro human prostate model. Med Biol Eng Comput, 37:618-624, 1999.

[32] Jennifer A Tuxhorn, Gustavo E Ayala, Megan J Smith, Vincent C Smith, Truong D Dang, and David R Rowley. Reactive stroma in human prostate cancer: induction of myofibroblast phenotype and extracellular matrix remodeling. Clin Cancer Res, 8:2912-2923, 2002.

[33] T. Oie, Y. Murayama, T. Fukuda, C. Nagai, S. Omata, K. Kanda, H. Yaku, and Y. Nakayama. Local elasticity imaging of vascular tissues using a tactile mapping system. J Artif Organs, 12:40-46, 2009.

[34] Leo Pallwein, Michael Mitterberger, Germar Pinggera, Friedrich Aigner, Florian Pedross, Johann Gradl, Alexandre Pelzer, Georg Bartsch, and Ferdinand Frauscher. Sonoelastography of the prostate: comparison with systematic biopsy findings in 492 
patients. Eur J Radiol, 65:304-310, 2008. 